\title{
Negotiation Among DDeLP Agents
}

\author{
Fernando A. Tohmé ${ }^{1}$ and Guillermo R. Simari ${ }^{2}$ \\ 1 Department of Economics and National Research Council (CONICET) \\ EMAIL: ftohme@criba.edu.ar \\ 2 Artificial Intelligence Research and Development Laboratory \\ Department of Computer Science and Engineering \\ EMAIL: grs@cs.uns.edu.ar \\ UniversidAd NACIONAL DEL SuR \\ Baha Blanca - ARGENTINA
}

\begin{abstract}
Negotiation can be conceived as the exchange of messages among self-interested agents in order to settle on an agreement over a given issue. They decide which messages to send according to their preferences and their evolving beliefs. Agents able to handle this dynamics of messages and beliefs can be represented by means of Defeasible Logic Programming augmented with utility functions. This approach to argumentation has the advantage of providing a useful platform for the representation of beliefs and the generation of messages. The interactive nature of negotiations requires an updating mechanism to be applied over the knowledge bases of the agents. The features of this mechanism are described by a protocol of a negotiation. Although there are many possible protocols, we concentrate on one that ensures the existence of an agreement in negotiations. The formalism of DeLP provides a very natural approach to the characterization of such a protocol.
\end{abstract}

\section{Introduction and Motivation}

The design of agents able to engage in negotiations is one of the main goals in the research on Multi-Agent Systems [LS01]. Justifications for the behavior of agents in negotiations have been known for a long time in disciplines related to the study of Decision-Making processes [Mye89]. Despite the efforts of many authors both in the Decision Sciences as in Multi-Agents Systems, the characterization of precise mechanisms of interaction in negotiations has shown to be a hard problem because, unlike markets and voting situations, the context of interaction varies from a negotiation to another. An abstract characterization of the conditions for mechanisms (protocols) that may ensure the convergence to agreements in negotiations has been presented in [Toh02]. We take up some of the ideas there, but adapted to the additional requirement of giving a precise foundation for the internal argumentation processes carried out by each individual agent.

To represent those deliberation processes we choose an alternative form of declarative programming, Defeasible Logic Programming (DeLP) [SCG94,Gar00,GS04]. This formalism combines Logic Programming 
with Defeasible Argumentation [Pol87,SL92,Pol95,Dun95,CML00,PV00], allowing the representation of tentative knowledge and leaving for the inference mechanism the task of finding the conclusions that the knowledge base warrants [CDSS03]. Furthermore, we introduce Decision-Theoretic tools into DeLP, in order to represent the fact that agents are self-interested. This is achieved by adding preferences to the formalism of DeLP, i.e. utility considerations [Lou90,TS04]. We call agents that reason using this Decision-theoretic enhanced Defeasible Logic Programming, DDeLP agents.

We consider only two-agent negotiations, since the extension to any number of agents follows basically the same pattern but is more involved in its syntax, without providing extra intuitions. In this simple kind of negotiations, one agent (by convention she is always called $\mathbf{a}_{1}$ ), chooses one preferred conclusion derived from her beliefs. This conclusion represents a possible settlement for the negotiation. The other agent $\left(\mathbf{a}_{2}\right)$ treats this message as a query $Q$. Agent $\mathbf{a}_{2}$ agrees with $\mathbf{a}_{1}$ if he can find a warranted argument $\mathcal{A}$ for $Q$. Otherwise, he explores his own knowledge base in order to select a new proposal to make.

If we want to ensure the convergence of the negotiation, we may impose over the agents a protocol to guide the process of exchange of messages towards an agreement [Lou98]. As shown in [Toh02] a sufficient condition for such a protocol is its monotonicity. One consequence of this property is the absence of cycles, understood as the repetition of messages. Since a message cannot be repeated by either of the agents, any attempt to "convince" the other party in the negotiation is implicitly ruled out. In fact, a monotonic protocol rather forces an agent to accept the message of the other as a constraint. ${ }^{3}$ Therefore, returning to our Decision-theoretic DeLP framework, if no warranted argument for the message of the other party is found, the agent must look for at least one rule responsible for this. From among several, she should choose the one that yields less utility and eliminate it from the knowledge base. Although there are other possible responses, this procedure represents a very cautious change of beliefs of the agent. With the corrected knowledge base, which represents the current beliefs of the agent, a message is chosen and the process repeats itself until either a query becomes warranted or there are no longer rules to be deleted from the knowledge base. In the first case the negotiation is said to end in an agreement. In the latter case, instead, it results in the breakup of the negotiation (which can be seen as a form of degenerate agreement on not pursuing further the negotiation).

The plan of the rest of this paper is as follows. In section 2 we will present the rudiments of DeLP with utilities. In section 3 we introduce the protocol and describe how it proceeds. Section 4 discusses possible extensions for this work.

\section{Decision-theoretic Defeasible Logic Programming}

We consider a language with three disjoint components:

\footnotetext{
${ }^{3}$ This is a requirement shared by all known models of bargaining.
} 
- Facts, which are ground literals representing atomic information (or the negation of atomic information).

- Strict Rules of the form $L_{0} \leftarrow L_{1}, \ldots, L_{n}$, where $L_{0}$ is the head and $\left\{L_{i}\right\}_{i>0}$ is the body. Each $L_{i}$ in the body or the head is a literal.

- Defeasible Rules of the form $L_{0} \prec L_{1}, \ldots, L_{n}$, where $L_{0}$ is the head and $\left\{L_{i}\right\}_{i>0}$ is the body. Each $L_{i}$ in the body or the head is a literal.

Then, a Defeasible Logic Program is a set of facts, strict rules, and defeasible rules. $\mathcal{P}=(\Pi, \Delta)$, where $\Pi$ denotes the set of facts and strict rules, while $\Delta$ denotes the set of defeasible rules. For each query $Q$ there are four possible answers: YES, NO, UNDECIDED or UNKNOWN.

To determine which answer is correct, we need the notion of argument. Given a program $\mathcal{P}=(\Pi, \Delta)$ and a literal $L,\langle\mathcal{A}, L\rangle$ is an argument structure for $L$. $\mathcal{A}$ is a set of defeasible rules in $\Delta$ such that:

1. there exists a defeasible derivation of $L$ from $\Pi \cup \mathcal{A}$. That is, there exists a finite sequence $L_{1}, \ldots, L_{n}=h$ of ground literals, such that each $L_{i}$ is either a fact in $\Pi$ or there exists a rule in $\Pi \cup \mathcal{A}$ with $L_{i}$ as its head, and every literal in the body $B_{j}$ is such that $B_{j} \in\left\{L_{k}\right\}_{k<i}$,

2. there is no literal $P$ such that both $P$ and $\neg P$ have defeasible derivations from $\Pi \cup \mathcal{A}$,

3. $\mathcal{A}$ is minimal, i.e., there does not exist $\mathcal{A}_{1} \subseteq \mathcal{A}$ such that $\mathcal{A}_{1}$ satisfies (1) and $(2)$.

This framework can be enhanced by means of preferences, $\Phi: \Pi \cup \Delta \rightarrow \mathbf{B}$, where $\mathbf{B}$ is an arbitrary Boolean algebra with top $\top$ and bottom $\perp$. The new elements $\Phi(\cdot)$ and $\mathbf{B}$ represent explicit preferences, in the sense that given two pieces of information $\mu_{1}, \mu_{2} \in \Pi \cup \Delta$ if $\mu_{1}$ is strictly more preferred than $\mu_{2}$ then $\Phi\left(\mu_{1}\right) \succ_{\mathbf{B}} \Phi\left(\mu_{2}\right)$, where $\succeq_{\mathbf{B}}$ is the order of $\mathbf{B}$. The elements of $\mu \in \Pi \cup \Delta$ which are most preferred receive a label $\Phi(\mu)=\top$.

We do not assume here that $\Phi$ assigns $\top$ to all strict rules in $\Pi$, and not even that $\Phi\left(\mu_{1}\right) \succ_{\mathbf{B}} \Phi\left(\mu_{2}\right)$ for $\mu_{1} \in \Pi$ and $\mu_{2} \in \Delta$. This is because $\Phi(\cdot)$ has, unlike the distinction between strict and defeasible rules, no epistemic content. Instead, the preferences represent other kinds of rationales. In particular the cost-benefit rates of the pieces of information (since their use may preclude the use of other pieces in the reasoning process).

Whatever the reasons are for preferring elements of $\Pi \cup \Delta$, we postulate a Boolean algebra $\mathbf{B}$ over which $\Phi(\cdot)$ ranges. It can be argued that a more general ordering could be appropriate but, as we will see, the inference engine has to perform some operations over the labels of the pieces of information used in the process of argumentation. In consequence, the range of $\Phi(\cdot)$ has to be not only an ordered set but also be closed under the logic operators $\Lambda$ and $\bigvee$. This can be easily represented by means of a Boolean algebra. In the simplest case, in which $\mathbf{B}$ is just a compact subset of real numbers with the natural order, we may say that $\Phi(\mu)$ is the utility of the piece of information $\mu$.

From the preferences over $\Pi \cup \Delta$, we can find preferential values over defeasible derivations. A fact $L$, which can be seen as the head of a (strict) rule 
with an empty body, has a value denoted $V(L, \emptyset)=\Phi(L)$. By induction, given rule $\mu$ (strict or defeasible) with head $L$ and body $B_{1}, \ldots, B_{m}$, if $L$ is derived using $\mu$ its preferential value is $V(L, \mu)=\Phi(\mu) \wedge \bigwedge_{k=1}^{m} V\left(B_{k}\right)$. The intuition here is that a conclusion is as strongly preferred as the weakest of either its premises or the rule used in the derivation. Given a defeasible derivation from $\Pi \cup \Delta, \mathcal{L}_{\Pi \cup \Delta}(L): L_{1}, \ldots, L_{n}=h$, it yields for its conclusion $L$ a value $V\left(h, \mathcal{L}_{\Pi \cup \Delta}(L)\right)=V(L, \mu)$ where $\mu$ is the rule that yields $h=L_{n}$ up from some literals in $\left\{L_{j}\right\}_{j<n}$.

By extension, an argument structure $\langle\mathcal{A}, L\rangle$ yields a value for $L, V(L, \mathcal{A})=$ $\bigwedge_{\mathcal{L}_{\Pi \cup \mathcal{A}}(L)} V\left(L, \mathcal{L}_{\Pi \cup \mathcal{A}}(L)\right)$. That is, it yields the lowest value among all the derivations of $L$ by using defeasible rules in $\mathcal{A}$. Notice that, by definition of $\mathcal{A}$ there is no other set $\mathcal{A}^{\prime} \subset \mathcal{A}$ that allows the derivation of $L$, but more than one selection of strict rules may exist in $\Pi$ that allows, jointly with $\mathcal{A}$, to do that.

Let $\mathcal{F}$ the set of all literals that can have a defeasible derivation from $\Pi \cup \Delta$. Any subset $H \subseteq \mathcal{F}$ has a value $V(H)=\bigvee_{L \in H} \bigwedge_{\mathcal{L}_{\Pi \cup \Delta}(L)} V\left(L, \mathcal{L}_{\Pi \cup \Delta}(L)\right)$. This means that $H$ is as valuable as the most valuable of its elements, which in turn is as valuable as the weakest of its derivations.

With this characterization we speak of an Decision-theoretic enhanced Defeasible Logic Program or $\mathcal{P}^{\prime}=(\Pi, \Delta, \Phi, \mathbf{B})$ which is intended to provide answers to queries through a process of argumentation that proceeds making comparisons among arguments. The main criterion of comparison used is preferential specificity [Poo85,SL92,SGCS03,TS04]. Consider a program $\mathcal{P}^{\prime}=(\Pi, \Delta, \Phi, \mathbf{B})$ with $\Pi_{G}$ the set of strict rules from $\Pi$. Let $\mathcal{F}$ the set of all literals that can have a defeasible derivation from $\Pi \cup \Delta$. Let $\left\langle\mathcal{A}_{1}, L_{1}\right\rangle$ and $\left\langle\mathcal{A}_{2}, L_{2}\right\rangle$ be two argument structures with $L_{1}, L_{2} \in \mathcal{F}$. Then $\left\langle\mathcal{A}_{1}, L_{1}\right\rangle$ is strictly more preferentially specific than $\left\langle\mathcal{A}_{2}, L_{2}\right\rangle$ if:

1. For all $H \subseteq \mathcal{F}$, if there exists a defeasible derivation of $L_{1}$ from $\Pi_{G} \cup H \cup \mathcal{A}_{1}$ while $\Pi_{G} \cup H \forall L_{1}$, then $L_{2}$ can be defeasibly derived from $\Pi_{G} \cup H \cup \mathcal{A}_{2}$, and

2. there exists $H^{\prime} \subseteq \mathcal{F}$ such that there exists a defeasible derivation of $h_{2}$ from $\Pi_{G} \cup H^{\prime} \cup \mathcal{A}_{2}$ and $\Pi_{G} \cup H^{\prime} \forall L_{2}$ but there is no defeasible derivation of $L_{1}$ from $\Pi_{G} \cup H^{\prime} \cup \mathcal{A}_{1}$.

3. For every $H$ verifying (1) and $H^{\prime}$ verifying (2), $V(H) \succeq_{\mathbf{B}} V\left(H^{\prime}\right)$.

Argument $\left\langle\mathcal{A}_{1}, L_{1}\right\rangle$ counterargues another $\left\langle\mathcal{A}_{2}, L_{2}\right\rangle$ at a literal $L$ if there exists a sub-argument of $\left\langle\mathcal{A}_{2}, L_{2}\right\rangle,\langle\mathcal{A}, L\rangle$, i.e., $\mathcal{A} \subseteq \mathcal{A}_{2}$, such that there exists a literal $P$ verifying both $\Pi \cup\left\{L, L_{1}\right\} \vdash P$ and $\Pi \cup\left\{L, L_{1}\right\} \vdash \neg P$.

If $\left\langle\mathcal{A}_{1}, L_{1}\right\rangle$ and $\left\langle\mathcal{A}_{2}, L_{2}\right\rangle$ are two argument structures, $\left\langle\mathcal{A}_{1}, L_{1}\right\rangle$ is a proper preferential defeater for $\left\langle\mathcal{A}_{2}, L_{2}\right\rangle$ at literal $L$ iff there exists a sub-argument of $\left\langle\mathcal{A}_{2}, L_{2}\right\rangle,\langle\mathcal{A}, L\rangle$ such that $\left\langle\mathcal{A}_{1}, L_{1}\right\rangle$ counterargues $\left\langle\mathcal{A}_{2}, L_{2}\right\rangle$ at $L$ and $\left\langle\mathcal{A}_{1}, L_{1}\right\rangle$ is strictly more preferentially specific than $\langle\mathcal{A}, L\rangle$. Alternatively, $\left\langle\mathcal{A}_{1}, L_{1}\right\rangle$ is a blocking preferential defeater for $\left\langle\mathcal{A}_{2}, L_{2}\right\rangle$ at literal $L$ iff there exists a sub-argument of $\left\langle\mathcal{A}_{2}, L_{2}\right\rangle,\langle\mathcal{A}, L\rangle$ such that $\left\langle\mathcal{A}_{1}, L_{1}\right\rangle$ counterargues $\left\langle\mathcal{A}_{2}, L_{2}\right\rangle$ at $L$ and neither $\left\langle\mathcal{A}_{1}, L_{1}\right\rangle$ is strictly more preferentially specific than $\langle\mathcal{A}, L\rangle$ nor is $\langle\mathcal{A}, L\rangle$ strictly 
more preferentially specific than $\langle\mathcal{A}, L\rangle$. If $\left\langle\mathcal{A}_{1}, L_{1}\right\rangle$ is either a proper or a blocking preferential defeater of $\left\langle\mathcal{A}_{2}, L_{2}\right\rangle$, it is said to be a preferential defeater of the latter.

An argumentation line for an argument structure $\left\langle\mathcal{A}_{0}, L_{0}\right\rangle$ is a sequence $\Gamma=\left[\left\langle\mathcal{A}_{0}, L_{0}\right\rangle,\left\langle\mathcal{A}_{1}, L_{1}\right\rangle,\left\langle\mathcal{A}_{2}, L_{2}\right\rangle, \cdots\right]$ where for each $i>0\left\langle\mathcal{A}_{i+1}, L_{i+1}\right\rangle$ is a defeater of $\left\langle\mathcal{A}_{i}, L_{i}\right\rangle . \Gamma_{S}=\left[\left\langle\mathcal{A}_{0}, L_{0}\right\rangle,\left\langle\mathcal{A}_{2}, L_{2}\right\rangle,\left\langle\mathcal{A}_{4}, L_{4}\right\rangle, \cdots\right]$ is the sequence of supporting argument structures of $\Gamma$, while the sequence of interfering ones is $\Gamma_{I}=\left[\left\langle\mathcal{A}_{1}, L_{1}\right\rangle,\left\langle\mathcal{A}_{3}, L_{3}\right\rangle,\left\langle\mathcal{A}_{5}, L_{5}\right\rangle, \cdots\right]$.

An acceptable argumentation line in a defeasible program $\mathcal{P}^{\prime}=(\Pi, \Delta, \Phi, \mathbf{B})$ is a finite sequence $\Gamma=\left[\left\langle\mathcal{A}_{0}, L_{0}\right\rangle, \cdots,\left\langle\mathcal{A}_{n}, L_{n}\right\rangle\right]$ such that:

1. Both $\Gamma_{S}$ and $\Gamma_{I}$ are concordant, i.e., there is no $P$ such that both $P$ and $\neg P$ have defeasible derivations from $\Pi \cup \bigcup_{i=0}^{\left\lfloor\frac{n}{2}\right\rfloor} \mathcal{A}_{2 i}$ and no $P^{\prime}$ with defeasible derivations for both $P^{\prime}$ and $\neg P^{\prime}$ from $\Pi \cup \bigcup_{i=0}^{\left\lfloor\frac{n-1}{2}\right\rfloor} \mathcal{A}_{2 i+1}$.

2. No argument $\left\langle\mathcal{A}_{k}, L_{k}\right\rangle \in \Gamma$ is a subargument of an argument $\left\langle\mathcal{A}_{j}, L_{j}\right\rangle$, i.e., $\mathcal{A}_{k} \not \subset \mathcal{A}_{j}$, for $j<k$.

3. For each $i<n$, if $\left\langle\mathcal{A}_{i}, L_{i}\right\rangle$ is a blocking preferential defeater of $\left\langle\mathcal{A}_{i-1}, L_{i-1}\right\rangle$ then $\left\langle\mathcal{A}_{i+1}, L_{i+1}\right\rangle$ is a proper preferential defeater of $\left\langle\mathcal{A}_{i}, L_{i}\right\rangle$.

To answer a query $Q$, the preferential warrant procedure builds up a candidate argument structure $\langle\mathcal{A}, Q\rangle$. Then, it associates to this argument a preferential dialectical tree $\mathcal{T}_{\langle\mathcal{A}, Q\rangle}$ as follows:

1. The root of the tree is labeled, $\left\langle\mathcal{A}_{0}, Q_{0}\right\rangle$, i.e., $\mathcal{A}_{0}=\mathcal{A}$ and $Q_{0}=Q$.

2. Let $n$ be a non-root node, with label $\left\langle\mathcal{A}_{n}, Q_{n}\right\rangle$ and $\Gamma=$ $\left[\left\langle\mathcal{A}_{0}, Q_{0}\right\rangle, \cdots,\left\langle\mathcal{A}_{n}, Q_{n}\right\rangle\right]$ the labels in the path from the root to $n$. Let $\mathcal{B}=\left\{\left\langle\mathcal{B}_{1}, H_{1}\right\rangle, \cdots,\left\langle\mathcal{B}_{k}, H_{k}\right\rangle\right\}$ be the set of all the preferential defeaters for $\left\langle\mathcal{A}_{n}, Q_{n}\right\rangle$. For $1 \leq i \leq k$, if $\Gamma^{\prime}=\left[\left\langle\mathcal{A}_{0}, Q_{0}\right\rangle, \cdots,\left\langle\mathcal{A}_{n}, Q_{n}\right\rangle,\left\langle\mathcal{B}_{i}, H_{i}\right\rangle\right]$ is an acceptable argumentation line, $n$ has a child $n_{i}$ labeled $\left\langle\mathcal{B}_{i}, H_{i}\right\rangle$. If $\mathcal{B}=\emptyset$ or no $\left\langle\mathcal{B}_{i}, H_{i}\right\rangle \in \mathcal{B}$ is such that $\Gamma^{\prime}$ is acceptable, then $n$ is a leaf of the tree.

The nodes of $\mathcal{T}_{\langle\mathcal{A}, Q\rangle}$ can be marked, yielding a tagged tree $\mathcal{T}_{\langle\mathcal{A}, Q\rangle}^{*}$ as follows:

- All leaves of $\mathcal{T}_{\langle\mathcal{A}, Q\rangle}$ are marked $U$ in $\mathcal{T}_{\langle\mathcal{A}, Q\rangle}^{*}$.

- If $\langle\mathcal{B}, H\rangle$ is the label of a node which is not a leaf, the node will be marked $U$ in $\mathcal{T}_{\langle\mathcal{A}, Q\rangle}^{*}$ if every child is marked $D$. Otherwise, if at least one of its children is marked $U$, it is marked as $D$.

Then, given an argument $\langle\mathcal{A}, Q\rangle$ and its associated tagged tree $\mathcal{T}_{\langle\mathcal{A}, Q\rangle}^{*}$, if the root is marked $U$, the literal $Q$ is said to be preferentially warranted. $\mathcal{A}$ is said to be the preferential warrant for $Q$. Therefore, given a query $Q$ the possible answers will be:

YES, $\quad$ if $Q$ is preferentially warranted;

NO, $\quad$ if $\neg Q$ is preferentially warranted;

UNDECIDED, if neither $Q$ nor $\neg Q$ are preferentially warranted;

UNKNOWN, if $Q$ is not in the language of the program. 


\section{Negotiation}

A negotiation can be seen as the exchange of messages among agents in order to reach an agreement over a given issue. The main elements in a negotiation are the following:

- The possible settlements.

- The preferences over them.

- The individual beliefs about the possible results.

- The messages that can be exchanged.

The messages are chosen according to the preferences over the class of settlements that are believed to be acceptable. Once a message is received, an agent has to decide whether to accept the implied settlement or to break up the negotiation or to explore for new possibilities. If the latter is the case, the agent has to update her beliefs and choose her message according to that.

In terms of DDeLP consider a program $\mathcal{P}^{\prime}=(\Pi, \Delta, \Phi, \mathbf{B})$ where $\Phi=\Phi_{1} \times$ $\ldots \Phi_{n}$ with range $\mathbf{B}^{n}$, from which we may define the elements of a $n$-person negotiation as follows:

- The possible settlements are the literals $L$ that can be defeasibly derived from $\Pi \cup \Delta$.

- The preferences over the literals are derived from $\Phi$.

- The individual beliefs are subsets of $\Pi \cup \Delta$.

- The messages are the literals $L$ plus two extra symbols, "yes" and "break", to indicate either agreement or the breakup of negotiations.

That is, we assume that agents consider only a certain subset of rules, from which they select some literals as both possible settlements and as messages. We consider only two agents, 1 and 2. Each agent $i$ performs, at each stage $t$ of the negotiation, a DDeLP program $\left\langle\Pi_{i}^{t}, \Delta_{i}^{t}, \Phi_{i}^{t}, \mathbf{B}\right\rangle$, where $\Pi_{i}^{t} \cup \Delta_{i}^{t} \subseteq \Pi \cup \Delta$ and $\Phi_{i}^{t}$ is the restriction of $\Phi_{i}$ over $\Pi_{i}^{t} \cup \Delta_{i}^{t}$.

At $t=1$ agent $\mathbf{a}_{1}$ sends a message (a literal $L^{1}$ derived from $\Pi_{1}^{1} \cup \Delta_{1}^{1}$ ) to agent $\mathbf{a}_{2}$. He may query his program $\Pi_{2}^{2} \cup \Delta_{2}^{2}$ and if the answer is YES end the negotiation by accepting $L$ and sending the message "yes". Otherwise, if the answer is UNKNOWN he may break the negotiation (because the issue has become meaningless) and send "break". In case that the answer is either NO or UNDECIDED, a new literal is chosen to be send as a message.

In general, a response to a message $L^{t}$ received by agent $\mathbf{a}_{i}$ at round $t+1$ of the negotiation, is a message $L^{t+1}$ defeasibly derived from $\Pi_{i}^{t+1} \cup \Delta_{i}^{t+1}$. The exchange of messages proceeds in orderly fashion: agent $\mathbf{a}_{1}$ sends her messages at odd values of $t$ (i.e. at $1,3, \ldots)$ while $\mathbf{a}_{2}$ sends hers at even values $(2,4, \ldots)$.

A possibility is that $L^{t}$ is either NO or UNDECIDED. Then, $\Pi_{i}^{t+1} \cup \Delta_{i}^{t+1}$ must be revised and updated according to $L^{t}$. The result of this operation of updating is $\bar{\Pi}_{i}^{t+1} \cup \bar{\Delta}_{i}^{t+14}$. There are alternative characterizations of this updating opera-

\footnotetext{
${ }^{4}$ The next time agent $i$ receives a message, $t+3$, his knowledge base will be $\Pi_{i}^{t+3} \cup$ $\Delta_{i}^{t+3} \equiv \bar{\Pi}_{i}^{t+1} \cup \bar{\Delta}_{i}^{t+1}$.
} 
tion [FKIS02], but a mandatory requirement is that it must be consistent with the protocol of negotiation.

Since we assume that the goal of both agents is either to reach an agreement or break up the negotiation, any sufficient condition that ensures such result may be applied to define a protocol. In [Toh02] it is shown that such condition is monotonicity, in the sense of reducing disagreements. In other words, agents are allowed to exchange messages (without repetition) until either an agreement is found or the negotiation breaks up.

In this sense, if until stage $t$ the messages that have been sent back and forth are $L^{1}, L^{2}, \ldots, L^{t-1}$, the possible messages to be send from then on are constrained by the protocol.

The following is obviously a monotonic protocol:

Protocol 31 If an agreement nor a break up is reached, the response to a message $L^{t}$ must be a $L^{t+1}$ in $\mathcal{L} \backslash\left\{L^{j}\right\}_{j=1}^{t}$, where $\mathcal{L}$ is the set of literals that can be defeasibly derived from $\Pi \cup \Delta$.

If such non-deterministic protocol is applied, a process of updating $\Pi_{i}^{t+1} \cup$ $\Delta_{i}^{t+1}$ must be consistent with it. In this sense, if $L^{t}$ is not preferentially warranted, no $L^{j}$ for $j=1, \ldots, t$ should be used as a message up from the resulting knowledge base $\bar{\Pi}_{i}^{t+1} \cup \bar{\Delta}_{i}^{t+1}$.

This means that, in particular, the current beliefs must be changed. The beliefs should no longer allow the messages sent in the previous rounds to be considered warranted. ${ }^{5}$ One way to achieve this is by using the following procedure:

Procedure 31 Consider, for a given $i$, the tagged trees $\mathcal{T}_{i}^{k}$, for $k=1, \ldots, t$. Among those with roots marked $U$, choose the leaves $\left\langle\mathcal{H}^{k}, H^{k}\right\rangle$ that minimize $V_{i}\left(H^{k}, \mathcal{H}^{k}\right)$, derived from $\Phi_{i}^{t+1}$.

For each of those leaves, choose a rule that minimizes $\Phi_{i}^{t+1}\left(\mu^{k}\right)$ over all the rules that participate in the derivation of $H^{k}$ from $\Pi^{t+1} \cup \mathcal{H}^{k}$. Then define $\bar{\Pi}_{i}^{t+1} \cup \bar{\Delta}_{i}^{t+1}:=\left(\Pi_{i}^{t+1} \cup \Delta_{i}^{t+1}\right) \backslash\{\mu\}_{k}$.

This procedure, used in the following algorithm allows to find the updated beliefs and choose next message:

Algorithm 31 [Update Beliefs and Select Message]

1. Define $\mathcal{T}_{\left\langle\mathcal{A}, L^{k}\right\rangle}$ for $k=1, \ldots, t$.

2. Run Procedure 3.1.

3. Find $\left\{\mathcal{T}_{i}^{k}\right\}_{k=1}^{t}$ over $\bar{\Pi}_{i}^{t+1} \cup \bar{\Delta}_{i}^{t+1}$

4. If a root is marked $U$ go to 2. Else

(a) If $\bar{\Pi}_{i}^{t+1} \cup \bar{\Delta}_{i}^{t+1}=\emptyset$, send the message "break".

${ }^{5}$ As said, preferences could also change, but this equivalent to replace $\Phi_{i}$ for $\Phi_{i}^{\prime}$. Although this can be easily introduced in our framework, we leave the details for an extension of this work. 
(b) Else choose $L^{t+1}$ such that minimizes $\Phi_{i}^{t+1}(L)$ over those literals $L$ that can be preferentially warranted in $\bar{\Pi}_{i}^{t+1} \cup \bar{\Delta}_{i}^{t+1}$.

It is immediate that:

Proposition 31 Algorithm 3.1 implements Protocol 3.1.

Proof: Trivial. If $L^{t}$ is warranted, then an agreement is reached and the selected message is "yes", else if $L^{t}$ is UNKNOWN the negotiation breaks up. Otherwise, Algorithm 3.1 is such that if $\bar{\Pi}_{i}^{t+1} \cup \bar{\Delta}_{i}^{t+1}=\emptyset$ the negotiation breaks up, otherwise, it ensures that $L^{1}, \ldots, L^{t}$ are not preferentially warranted in $\bar{\Pi}_{i}^{t+1} \cup \bar{\Delta}_{i}^{t+1}$, therefore it chooses $L^{t+1} \in \mathcal{L} \backslash\left\{L^{j}\right\}_{j=1}^{t}$.

Notice that Algorithm 3.1 is not the only possible implementation of Protocol 3.1, since the latter just asks for monotonicity in the messages, while the Algorithm intends to find the best messages for agent $\mathbf{a}_{i}$.

Another result that follows is:

Proposition 32 If both agents use Algorithm 3.1 to choose messages, the negotiation either ends in an agreement or in a break up.

Proof: Algorithm 3.1 implements Protocol 3.1. We denote $\mathcal{L}^{t}=\mathcal{L} \backslash\left\{L^{k}\right\}_{k=1}^{t}$. There are two possibilities, either there exists a $T$ such that when one of the agents sends a message $L^{T}$ the other agent responds with "yes". Alternatively, if there is no $L$ that may result in an agreement, as a consequence of the Compactness Theorem for First-Order Logic there exists a $T^{\prime}<\infty$ such that $\mathcal{L}^{T^{\prime}}=\emptyset$ and leads to a response "break".

A final consequence of using Algorithm 3.1 is that if agreements (including break ups as degenerate agreements) are path dependent. That is, the choices made by the agents condition further choices. Therefore, over the same knowledge base, the agents may end up agreeing on different conclusions. The following example shows this:

Example 31 Consider the classical example in defeasible argumentation where preferences are defined for $\mathbf{B}=\{0,1\}$, with $0<1$. The preferences, which for simplicity are assumed common to both agents, are indicated in parentheses next to the corresponding pieces of information:

$\Pi=\{\operatorname{bird}(X) \prec$ penguin $(X)(1)$, penguin(tweety) (1), bird(tweety) (1) $\}$

$\Delta=\{\neg$ flies $(X) \prec \operatorname{penguin}(X)(1)$, flies $(X) \prec \operatorname{bird}(X)(0.5)\}$

Agents have different beliefs:

$\Pi_{1}^{1}=\{$ penguin(tweety) (1), bird(tweety) $(1)\}$

$\Delta_{1}^{1}=\{\neg$ flies $(X) \prec \operatorname{penguin}(X)(1)\}$ 
while

$\Pi_{2}^{1}=\{\operatorname{bird}(X) \prec \operatorname{penguin}(X)(1)$, penguin(tweety) $(1)$,

$\Delta_{2}^{1}=\{\operatorname{flies}(X) \prec \operatorname{bird}(X)(0.5)\}$

For agent $\boldsymbol{a}_{1}$, there are two warranted conclusions, penguin(tweety) and $\neg$ flies(tweety). Suppose that her message is $L^{1}=$ penguin(tweety). Since it is also a warranted conclusion for $\boldsymbol{a}_{2}$, he will respond with "yes". Otherwise, suppose that $L^{1}=\neg$ flies(tweety). Since for $\boldsymbol{a}_{1}$ flies(tweety) is warranted he must apply Algorithm 3.1. It follows that he has to drop the rule with lowest preference, namely flies $(X) \prec \operatorname{bird}(X)$. Then,

$\Pi_{2}^{2}=\{\operatorname{bird}(X) \prec \operatorname{penguin}(X)(1), \operatorname{penguin}($ tweety $)(1)$,

$\Delta_{2}^{2}=\emptyset$

The only (trivially) warranted conclusions are penguin(tweety) and bird(tweety). In the case that $L^{2}=$ penguin(tweety), the response is "yes" and the same is true if $L^{2}=\operatorname{bird}($ tweety).

On the other hand, assume that $\Phi($ flies $(X) \prec \operatorname{bird}(X))=1$ while $\Phi(\operatorname{bird}(X) \prec \operatorname{penguin}(X))=0.5$. Accordingly, if $L^{1}=\neg$ flies(tweety) then

$\Pi_{2}^{2}=\{$ penguin(tweety) $(1)$,

$\Delta_{2}^{2}=\{\operatorname{flies}(X) \prec \operatorname{bird}(X)(1)\}$

Therefore, $L^{2}=$ penguin(tweety), and the response is "yes".

Finally, if

$\Pi_{2}^{1}=\{$ bird(tweety) $(1)\}$

$\Delta_{2}^{1}=\{\operatorname{flies}(X) \prec \operatorname{bird}(X)(0.5)\}$

and $L^{1}=$ penguin(tweety), the response is "break".

\section{Conclusions}

We presented in this paper a framework of negotiation with DeLP extended with preferences. An algorithm of belief updating based on the elimination of rules ensures that agents will reach an agreement, although this agreement depends on the particular sequence of messages chosen.

A matter of further work will be to see if a negotiation still converges to an agreement if another mechanism of belief updating is used. 


\section{Acknowledgments}

This research was partially supported by CONICET, by the Secretaría General de Ciencia y Tecnología de la Universidad Nacional del Sur and by Agencia Nacional de Promoción Científica y Tecnológica (PICT 2002 No. 13096). The authors would like to thank anonymous reviewers for providing helpful comments to improve the final version of this paper.

\section{References}

[CDSS03] Carlos I. Chesñevar, Jürgen Dix, Frieder Stolzenburg, and Guillermo R. Simari. Relating defeasible and normal logic programming through transformation properties. Theoretical Computer Science, 290(1):499-529, Jan 2003.

[CML00] Carlos I. Chesñevar, Ana G. Maguitman, and Ronald P. Loui. Logical Models of Argument. ACM Computing Surveys, 32(4), December 2000.

[Dun95] Phan M. Dung. On the acceptability of arguments and its fundamental role in nonmonotonic reasoning and logic programming and $n$-person games. Artificial Intelligence, 77:321-357, 1995.

[FKIS02] Marcelo A. Falappa, Gabrielle Kern-Isberner, and Guillermo R. Simari. Explanations, belief revision and defeasible reasoning. Artificial Intelligence Journal, 141(1-2):1-28, October 2002.

[Gar00] Alejandro J. García. Defeasible Logic Programming: Definition, Operational Semantics and Parallelism. PhD thesis, Computer Science and Engineering Department, Universidad Nacional del Sur, Bahía Blanca, Argentina, December 2000.

[GS04] Alejandro J. García and Guillermo R. Simari. Defeasible logic programming: An argumentative approach. Theory and Practice of Logic Programming, 4(1):95-138, 2004.

[KSE98] Sarit Kraus, Katia Sycara, and Amir Evenchik. Reaching agreements through argumentation: A logical model and implementation. Artificial Intelligence, 104(1-2):1-69, 1998.

[Lou90] Ronald P. Loui. Defeasible specification of utilities. In Henry Kyburg, Ronald Loui, and Greg Carlson, editors, Knowledge Representation and Defeasible Reasoning, pages 345-359. Kluwer Academic Publishers, Dordrecht, 1990.

[Lou98] Ronald P. Loui. Process and policy: Resource-bounded nondemonstrative reasoning. Computational Intelligence: An International Journal, 14, 1998.

[LS01] Kate Larson and Tuomas Sandholm. Bargaining with limited computation: Deliberation equilibrium. Artificial Intelligence, 132, 2001.

[Mye89] Roger B. Myerson. Credible negotiation statements and coherent plans. Journal of Economic Theory, 48, 1989.

[Pol87] John Pollock. Defeasible Reasoning. Cognitive Science, 11:481-518, 1987.

[Pol95] John Pollock. Cognitive Carpentry: A Blueprint for How to Build a Person. MIT Press, 1995.

[Poo85] David L. Poole. On the Comparison of Theories: Preferring the Most Specific Explanation. In Proc. 9th IJCAI, pages 144-147. IJCAI, 1985.

[PV00] Henry Prakken and Gerard Vreeswijk. Logical systems for defeasible argumentation. In D.Gabbay, editor, Handbook of Philosophical Logic, 2nd ed. Kluwer Academic Pub., 2000. 
[SCG94] Guillermo R. Simari, Carlos I. Chesñevar, and Alejandro J. García. The role of dialectics in defeasible argumentation. In XIV International Conference of the Chilenean Computer Science Society, November 1994.

[SGCS03] Frieder Stolzenburg, Alejandro J. García, Carlos I. Chesñevar, and Guillermo R. Simari. Computing generalized specificity. Journal of Aplied Non-Classical Logics, 13(1):87-113, January 2003.

[SL92] Guillermo R. Simari and Ronald P. Loui. A Mathematical Treatment of Defeasible Reasoning and its Implementation. Artificial Intelligence, 53:125157, 1992.

[Syc89] Katia Sycara. Multi-agent compromise via negotiation. In L. Gasser and M. Huhns, editors, Distributed Artificial Intelligence (Vol. 2). Morgan Kaufmann, Los Altos, CA, September 1989.

[Syc90] Katia Sycara. Persuasive argumentation in negotiation. Theory and Decision, 28(3):203-242, May 1990.

[Toh02] Fernando Tohmé. Negotiation and defeasible decision making. Theory and Decision, 53(4):289-311, 2002.

[TS04] Fernando A. Tohmé and Guillermo R. Simari. Preferential defeasibility: Utility in defeasible logic programming. In Proceedings of the 10th International Workshop on Non-Monotonic Reasoning, pages 394-399. NMR, June 2004. 